\title{
Improved confinement and related physics study in Compact Helical System
}

\author{
S. Okamura, T. Akiyama, A. Fujisawa, K. Ida, H. Iguchi, M. Isobe, S. Kado ${ }^{1)}$, \\ T. Minami, K. Nagaoka, K. Nakamura, S. Nishimura, K. Matsuoka, \\ H. Matsushita, H. Nakano, S. Ohshima, T. Oishi ${ }^{2)}$, A. Shimizu, C. Suzuki, \\ C. Takahashi, K. Toi, Y. Yoshimura, M. Yoshinuma and CHS group
}

National Institute for Fusion Science, Oroshi 322-6, Toki 509-5292, Japan

1) High Temperature Plasma Center, The University of Tokyo, 5-1-5 Kashiwanoha, Kashiwa, Chiba 227-8568 Japan

2) Department of Quantum Engineering and System Science, Graduate School of Engineering, The University of Tokyo, Hongo 7-3-1, Tokyo 113-8656 Japan

\section{Introduction}

Finding improved confinement modes is one of very important research objectives in magnetic confinement experiments of fusion research. In addition, the understanding of physical mechanism of improved confinement is necessary to establish a reliable operation of high confinement discharges and to extend those improved performances towards more attrac-tive scenarios of fusion reactor. CHS (Compact Helical System) experiments have been demonstrating various types of confinement improved modes in stellarator plasmas.

The internal transport barrier (ITB) for ECH plasmas was found in CHS for the first time in stellarator research [1]. The plasma potential profile and the local fluctuations were measured precisely by the heavy ion beam probe (HIBP) and it was confirmed that the mech-anism of the transport reduction was due to the electric field shear created by the neo-classical non-ambipolar flow of electrons and ions. Because of such a mechanism of creating the electric field shear, we call the ITB in CHS as a neo-classical ITB (N-ITB) in comparison to the ITB formation observed in tokamaks. After the observation in CHS, the ITB formations for electrons have been found in other stellarators (W7-AS, LHD, TJ-II). The ITB research in CHS progressed to the experiments with the combined heating of ECH and NBI. It was found that the ion temperature increased making the temperature pedestal when the ITB for electrons was created [2]. This new type of ITB formation for both electrons and ions is observed only in CHS and has never been found in other stellarators.

Even though ITB is very effective to increase the central temperature, an improvement of the global confinement is not large because of the small volume of the central part of plasma. From this point of view, formations of the edge transport barrier (ETB) have been also studied in CHS with NBI heated plasmas. H-mode discharges with ETB in stellarator were first found in CHS [3] simultaneously with Wendelstein7-AS [4] in 1996. It is com-mon features in the transport barrier formation that the location of the transport barrier is strongly related to the local rotational transform value (low order rationals are important). In the H-mode study in CHS at that time, the generation of inductive current using the ramp-up 
of the poloidal coil current was used for the control of the edge rotational transform. Those constraints in the operation limited the operational and diagnostic flexibilities of CHS for further studies of ETB physics. Five years ago, the NBI arrangement of CHS was changed from a balanced arrangement (co- and counter-injection) to the one with both in co-injection to avoid the low heating efficiency with counter-injection due to the orbit loss of high energy ions in a small plasma size. Together with the efforts of the vacuum wall conditioning, we found the new type of H-mode discharges with a clear ETB formation [5, 6]. We report in this paper general characteristics of $\mathrm{H}$-mode discharges in $\mathrm{CHS}$ and results of local measurements for the plasma edge region.

For the plasma confinement study in general, measurements of plasma turbulence is very important because it is well known that the anomalous transport is a dominant mecha-nism for any type of confinement devices for fusion research. The physical mechanism of the transport barrier related to the improved confinement is better understood when the tur-bulence is measured at the barrier region. The local measurement of fluctuations and the spatial coherence study are necessary for the quantitative understanding of the transport. The HIBP is the most powerful diagnostics for the turbulence study because it can give a local measurement of the plasma potential fluctuations with very high time response (up to $100 \mathrm{kHz}$ ). Since HIBP in CHS has three simultaneous measuring positions with about $1 \mathrm{~cm}$ distance, the coherence study of fluctuations with a scale length of $1 \mathrm{~cm}$ is also possible. In CHS, two HIBPs are installed at two toroidal locations with 90 degrees distance in the torus. With their almost identical specifications, various advanced measurements of fluctuations have become possible including a long range coherence measurement. It is very unique characteristics of CHS experiment and there is no other experiment in the world where we can conduct this type of advanced study of plasma confinement. In this paper, selected topics of turbulence measurements with duo HIBPs will be reported related to the zonal flow and the transport reduction with the ITB formation.

\section{Edge Transport Barrier Formation}

Figure 1 shows time traces of plasma global parameters showing the transition to the ETB formation and the back transition. Two neutral beams are injected into a low density hydrogen plasma created by the ECH heating $(53 \mathrm{GHz}, 200 \mathrm{~kW})$. Beam energy for two NBIs is 40 and $30 \mathrm{keV}$, respectively, and the maximum port-through injection power is about $800 \mathrm{~kW}$ for each. The standard magnetic configuration in CHS was used with the magnetic axis position $R_{a x}=0.92 \mathrm{~m}$. The magnetic field strength is $0.95 \mathrm{~T}$ at the magnetic axis. As shown in Fig. 1, the plasma is sustained by NBI and density increases with gas puffing. After $35 \mathrm{msec}$ from the start of NBI heating, a spontaneous transition appears in the plasma edge region which is clearly shown by the sharp drop of $\mathrm{H} \alpha$ emission signal. The estimated NBI deposition power is about 0.6 MW before the transition. The delay time between the start of NBI heating and the transition largely depends on the magnetic configuration and the heating power, which will be discussed later. Figure 1 shows two chord signals of HCN interferometer at the center and at the normalized minor radius of 0.63 . These data show that the density profile becomes slightly peaked during the NBI heating phase before the transition with the edge density staying almost constant. After the transition at $78 \mathrm{msec}$, the 
edge density starts to increase making a flatter profile and the diamagnetic plasma energy increases as well by about $40 \%$. Volume averaged beta is $0.6 \%$ after the transition. The total radiation loss from the plasma also starts to increase. When the NBI.\#2 is turned off and the heating power is decreased, the back transition appears. The plasma current is a combination of the bootstrap current and the beam driven current. The current level of $8 \mathrm{kA}$ in this discharge gives small increase of the edge rotational transform (about 3\%) above the vacuum value created by the helical coils. It is not larger than the ambiguity of the edge rotational transform value given from the equilibrium calculation.
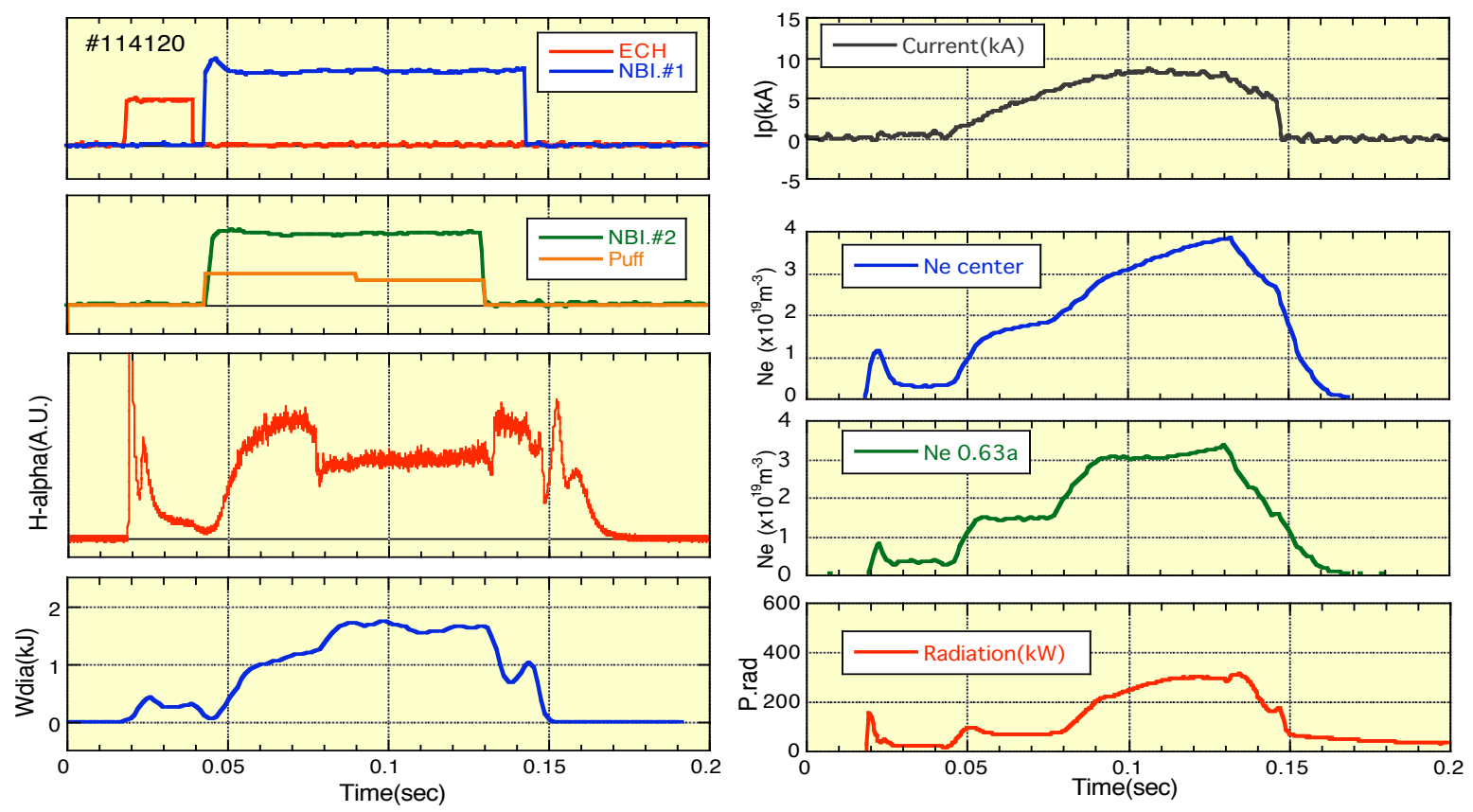

Fig. 1 Time traces of basic plasma parameters for NBI discharge with ETB formation. In left column are: time sequences of $\mathrm{ECH}, \mathrm{NBI}$ and gas puffing, $\mathrm{H} \alpha$ emission and diamagnetic energy. In right column are: plasma current, line averaged electron density for central chord, for edge chord and total radiation power.

The change of density profile at the transition was measured by the YAG Thomson scat-tering system in CHS which gives full profiles of electron temperature and density at every $5 \mathrm{msec}$. Figure 2 shows measured profiles of the discharge shown in Fig. 1 at $75 \mathrm{msec}$ (just before the transition) and $80 \mathrm{msec}$ (after the transition). The edge density increases after the transition making the larger density gradient at the plasma edge. Density profiles at two timings are compared with $\mathrm{HCN}$ interferometer signals for two measuring chords and these data are consistent with each other. The temperature profiles do not show notable difference with ETB formation for this discharge. However it does not mean that the improvement of the electron heat transport is negligible. Because of the density increase at the edge (and little change in the temperature gradient), the effective electron heat transport coefficient should be reduced with the ETB formation. 

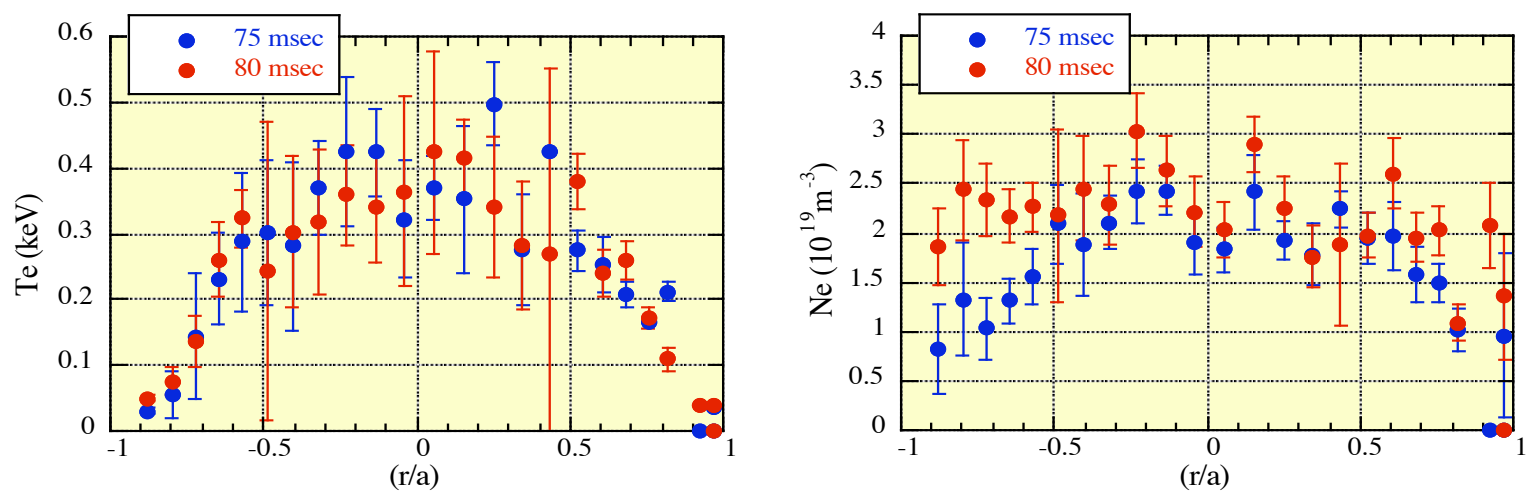

Fig. 2 YAG Thomson scattering measurements for electron temperature (left) and density (right) profiles. Blue point profile is before the transition and red one is after the transition.

\section{Power Threshold for ETB Formation}

Heating power threshold clearly exists for the ETB formation in CHS [7]. Figure 3 (a) shows the calculated absorbed power as a function of the line-averaged density for various discharges with the heating power close to the threshold. The magnetic configuration is the standard one with $\mathrm{R}_{\mathrm{ax}}=0.92 \mathrm{~m}$. Closed circles are for discharges with clear drop of $\mathrm{H \alpha}$ signal and open circles for discharges without Ha drop. Discharges with brown circles have dithering motion of $\mathrm{H \alpha}$ signal which indicates switching between $\mathrm{H}$ and $\mathrm{L}$ phase. The threshold power is increasing function of the density with a power of $0.4\left(\mathrm{ne}^{0.4}\right)$. The dependence of power threshold on the magnetic field strength was studied in the range $0.8 \mathrm{~T}$ $<\mathrm{Bt}<1.3 \mathrm{~T}$. In plotting Fig. 3 (b), we calculated the heating power normalized to the density based on the density dependence of the threshold power given in Fig. 3 (a). It is almost proportional to the magnetic field. The power threshold obtained in CHS experiments can be compared with the tokamak H-mode threshold scaling because it consists only of the density, magnetic field and device size parameters. The power threshold obtained in the discharges shown in Fig. 3 is roughly two times larger than tokamak scaling.
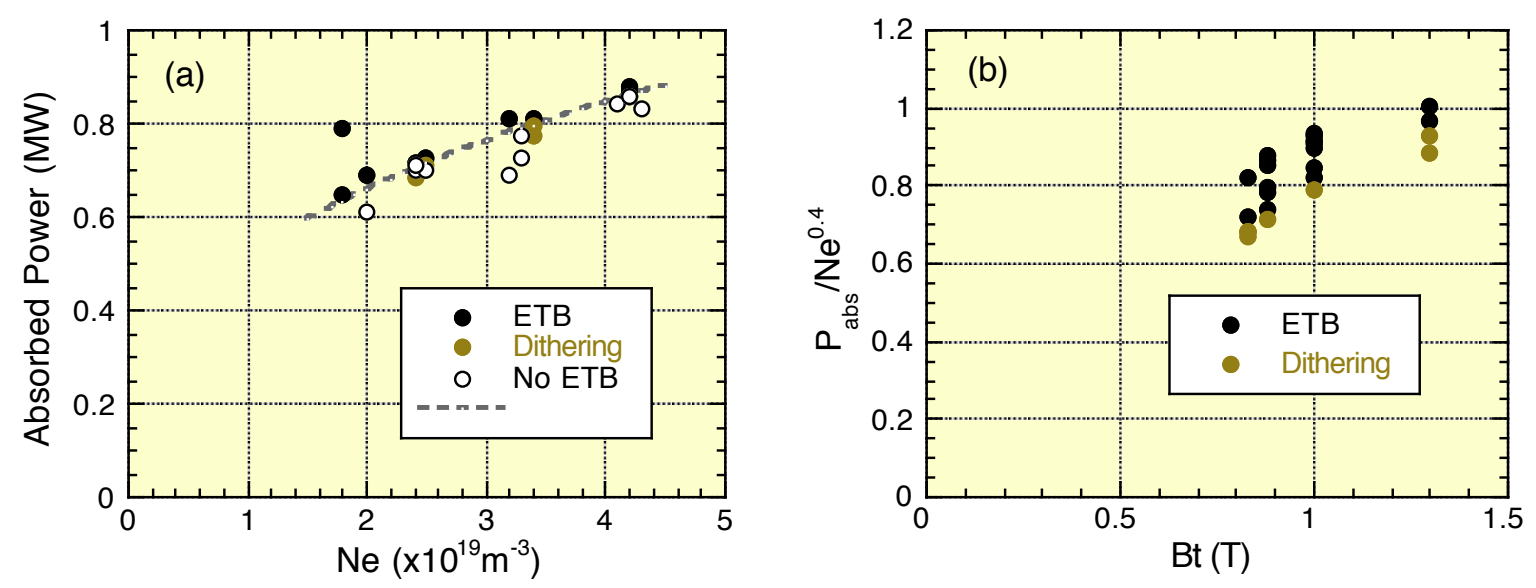

Fig. 3 Dependence of heating power threshold of ETB discharges on density (a) and on magnetic field strength (b). Absorbed power is normalized to the density in figure (b). 
H-mode power threshold in CHS depends also on the magnetic field configuration. Power threshold data shown in Fig. 3 were obtained for the configuration with the magnetic axis position at $0.92 \mathrm{~m}$. Figure 4 (a) shows the absorbed power normalized by the density for ETB discharges for various magnetic configurations with different magnetic axis positions $\mathrm{R}_{\mathrm{ax}}$. Because the density value is taken at the transition timing, discharges without transition are not plotted in the figure. Minimum value of normalized power gives the power threshold for each configuration. When the plasma position is shifted outward in torus, the power threshold for the transition decreases. H-mode transition is observed only for configurations with magnetic axis position in the range: $0.88 \mathrm{~m}<\mathrm{R}_{\mathrm{ax}}<0.95 \mathrm{~m}$. The power threshold for $\mathrm{R}_{\mathrm{ax}}=0.935 \mathrm{~m}$ configuration is almost comparable to the power threshold for tokamak H-mode discharges.
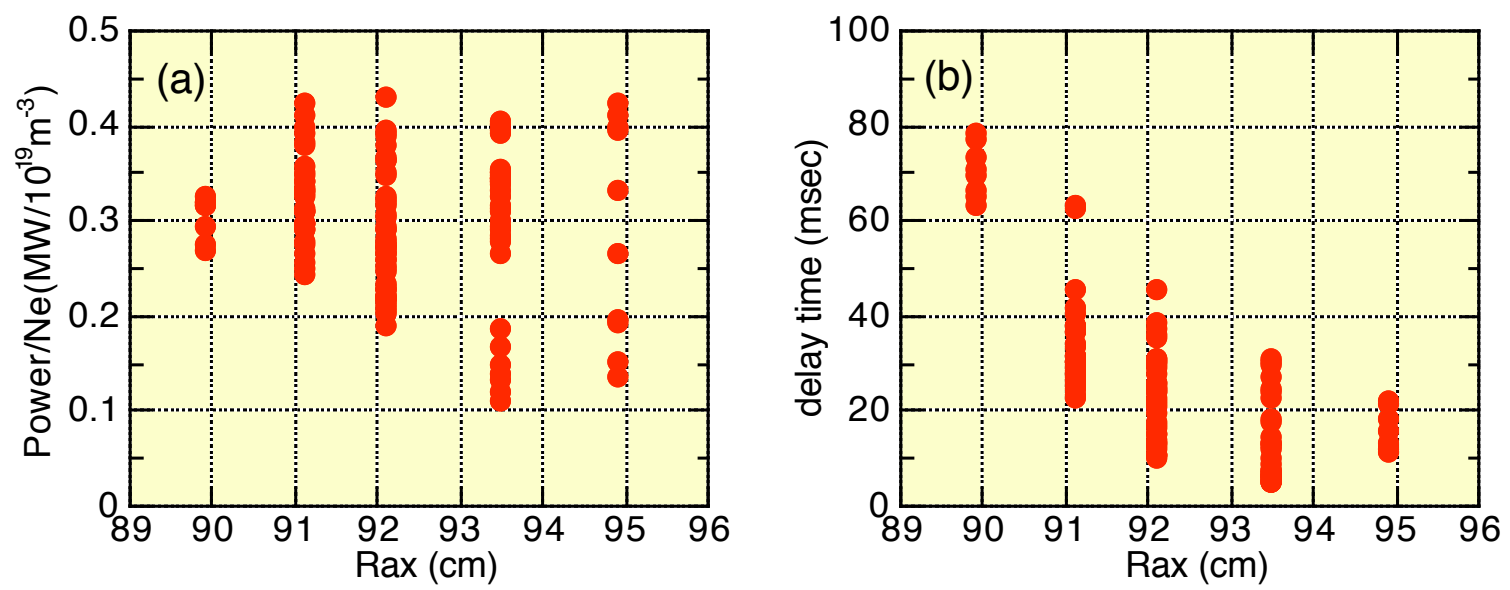

Fig. 4 (a) Dependence of power threshold of ETB discharge on magnetic configuration. (b) Dependence of delay time of transition after starting of NBI heating in the function of $\mathrm{R}_{\mathrm{ax}}$.

Among many magnetic surface quantities that vary depending on the magnetic axis position $\mathrm{R}_{\mathrm{ax}}$, the rotational transform at the plasma edge is the most important key parameter for the ETB formation. The profile of the rotational transform of CHS has a strong stel-larator type shear at the plasma edge. It is about $0.3(=1 / q)$ at the magnetic axis and slightly higher than 1 at the plasma edge. The edge rotational transform increases as $\mathrm{R}_{\mathrm{ax}}$ increases in the range of Fig. 4 (a). In other words, the position of the location of the unity rotational transform moves in when $\mathrm{R}_{\mathrm{ax}}$ is increased. Fig. 4 (a) indicates that the ETB is formed with smaller heating power when the unity rotational transform surface is sufficiently inside the plasma edge. Figure 4 (b) shows the delay time of spontaneous transition of ETB formation after the starting of NBI heating. This delay time also depends strongly on the magnetic configuration. One possible reason for this delay is the gradual change of the equilibrium with the increase of plasma beta. For example, the edge rotational transform of $\mathrm{R}_{\mathrm{ax}}=0.90 \mathrm{~m}$ configuration is lower than unity for the vacuum equilibrium and its value increases above unity with higher beta. After this delay time, the transition to ETB might occur with the unity rotational transform located within a plasma edge.

\section{Local Density Fluctuation Measurement}


A powerful diagnostics for the local density measurement in CHS is the beam emission spectroscopy (BES) which measures $\mathrm{H} \alpha$ emission of high energy hydrogen beam of NBI system excited by bulk plasma electrons [8]. NBI.\#2 is used for the beam source for BES measurement. An eight channel array of visible detectors is installed for obtaining the spa-tial density profile in the plasma boundary region. Since this system has a high frequency response $(<100 \mathrm{kHz})$, local fluctuation measurement is also possible.

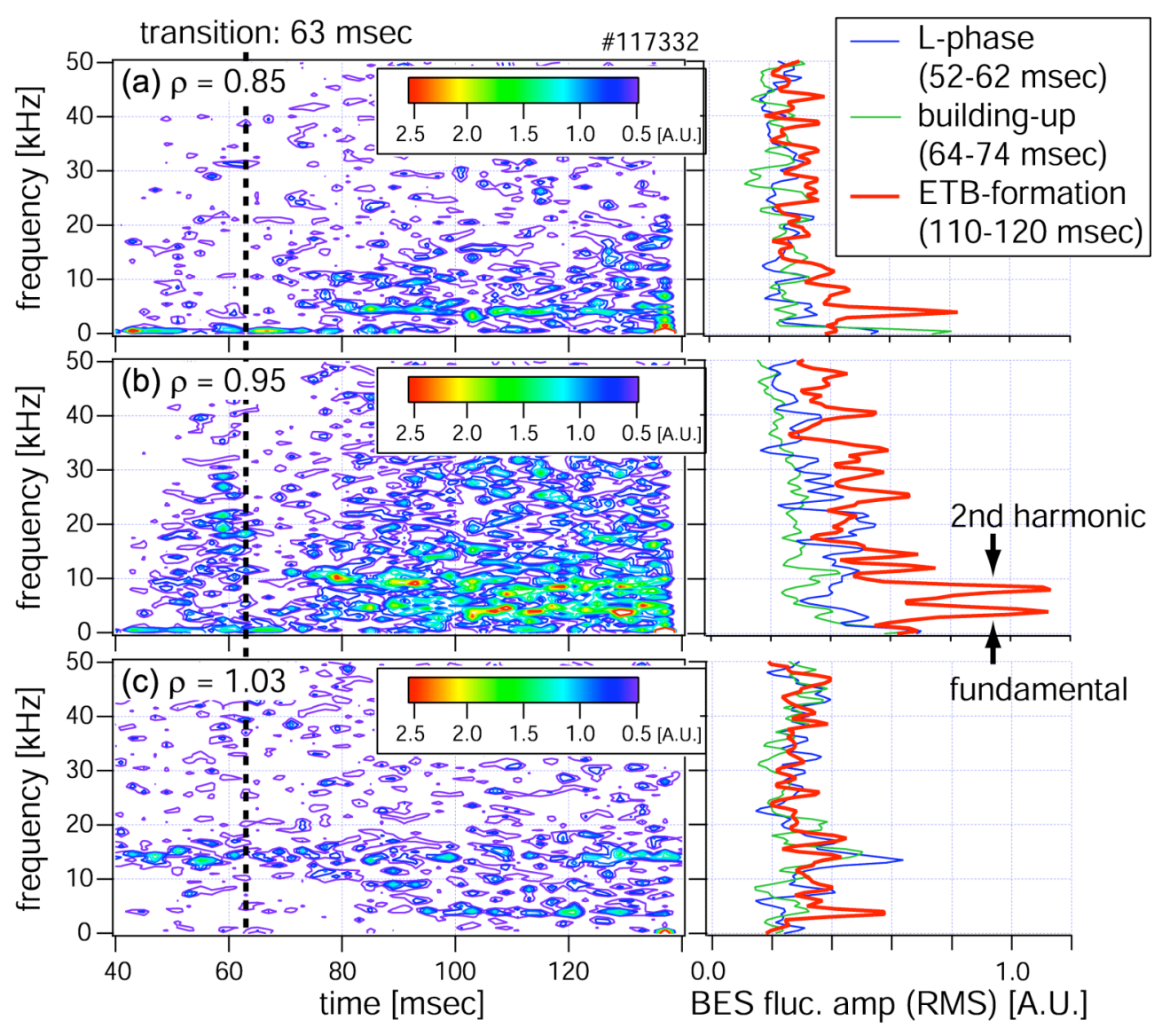

Fig. 5 Power spectra of local density fluctuations measured by BES. Evolution of spectra after ETB transition are shown for three measuring channel near plasma boundary. Cross sections of spectrum amplitude are shown at right for three timings: before transition, just after transition and at saturated phase of density pedestal.

When the heating power is much higher than the threshold, strong coherent fluctuations were detected by magnetic probes during the later phase of ETB formation when the edge density increase was saturated. A typical fundamental frequency is $4 \mathrm{kHz}$ and a couple of its harmonics also appeared. These characteristics are very similar to the edge harmonic oscil-lations (EHO) observed in DIII-D tokamak. In order to study the local structure of those oscillations, spectra of BES signals were analyzed for three channels near plasma edge. Figure 5 shows time evolution of the power spectrum with different color showing amplitude level (red for higher amplitude) [9]. The ETB transition occurred at $63 \mathrm{msec}$ and the edge density increased for the time period from 63 to $90 \mathrm{msec}$. After $90 \mathrm{msec}$, the edge density stayed at almost constant although the central density continued to increase making more peaked density profile in the later phase of ETB discharge. 
Cross sections of spectra are shown on the right side of figure to compare three time periods: before the transition, just after the transition and in the edge density saturation phase. Coherent fluctuations with the same frequency as magnetic probes appeared in the late phase of ETB discharge for three channels and the amplitude is much larger at the normalized radius $\rho=0.95$. At this radius, the second harmonic oscillation is clearly detected. It is shown also by the BES diagnostics that the density gradient is largest at this radius. A time of appearance of EHO correlates to the completion of the high density gradient and, after EHO appears, the edge density increase is saturated. This fact can be a qualitative evidence that the EHO in CHS enhances the outward particle flux to sustain the stable edge density pedestal.

\section{Fluctuation Measurements with Duo HIBPs}

In the plasma turbulence measurements for the plasma confinement study, the zonal flow is one of highly topical research subjects. In order to identify the zonal flow, it is important to reveal its global coherent characteristics and spatial structure. Duo HIBPs in CHS are a unique diagnostics in the world to answer those requests. The zonal flow has two branches of spectrum, i.e., a low frequency mode and the geodesic acoustic mode (GAM) with higher frequency. We already reported the identification of low frequency zonal flow ( $\mathrm{f}<10 \mathrm{kHz}$ ) [10]. In this paper, measurements of $10 \mathrm{kHz}$ range coherent fluctuations will be discussed which have characteristics of GAM.
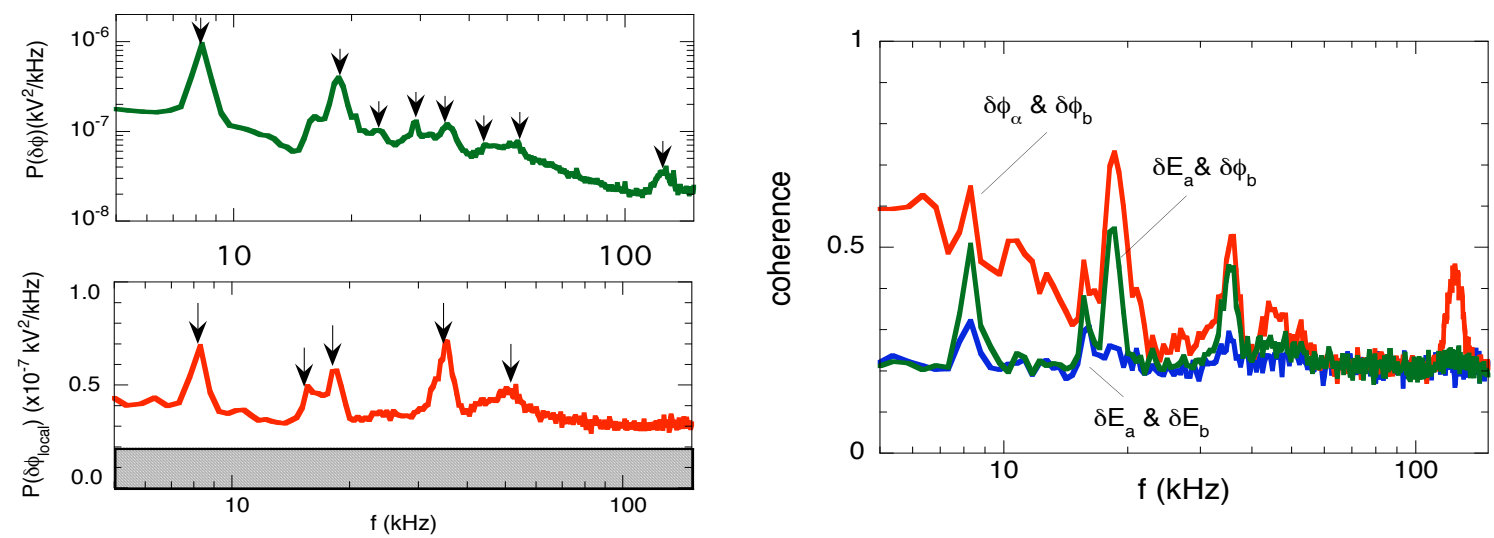

Fig. 6 Power spectra of potential fluctuation (left-top) and local electric field fluctuation (left-bottom). Coherence between two HIBP signals for three pairs of potential and electric field fluctuations (right).

Figure 6 (left) shows the power spectra of potential fluctuations in the frequency range of $10 \mathrm{kHz}$. Upper left spectrum is for the potential signal of single HIBP measurement. It contains both local potential signal and the effect of potential oscillation along the path of HIBP ion beam. Many peaks appear in the range of GAM frequency corresponding to the plasma parameters of the experiment. Bottom left figure shows the power spectrum of difference of potentials between two adjacent measuring point of single HIBP. Such a difference signal gives the local electric field. Because two ion beams for these detectors path through almost the same plasma area, the effect of potential oscillation along the beam path is cancelled out, the effect of which is demonstrated as eliminations of some peaks 
appearing in the potential spectrum. The coherence between these signals is shown in the right figure. Strong coherence is detected for the potential signal of two HIBPs. In order to confirm that the potential fluctuations are actually local ones instead of global oscillation, a coherence between local electric field is calculated. Although the coherence value is rela-tively small due to insufficient signal to noise ratio, a couple of peaks are identified which is presumably GAM oscillations.

Finally an example of turbulent particle flow measurement by HIBP is shown. Figure 7 shows the time evolution of the spectrum of turbulent particle flow calculated with HIBP signals for the local density and electric field fluctuations and their coherence and phase difference. This measurement was made for the ITB discharge where the transport barrier disappeared (back transition) at $72 \mathrm{msec}$. After the back transition, it is clear that the turbulent particle flow is enhanced for the frequency range of 50 to $100 \mathrm{kHz}$.

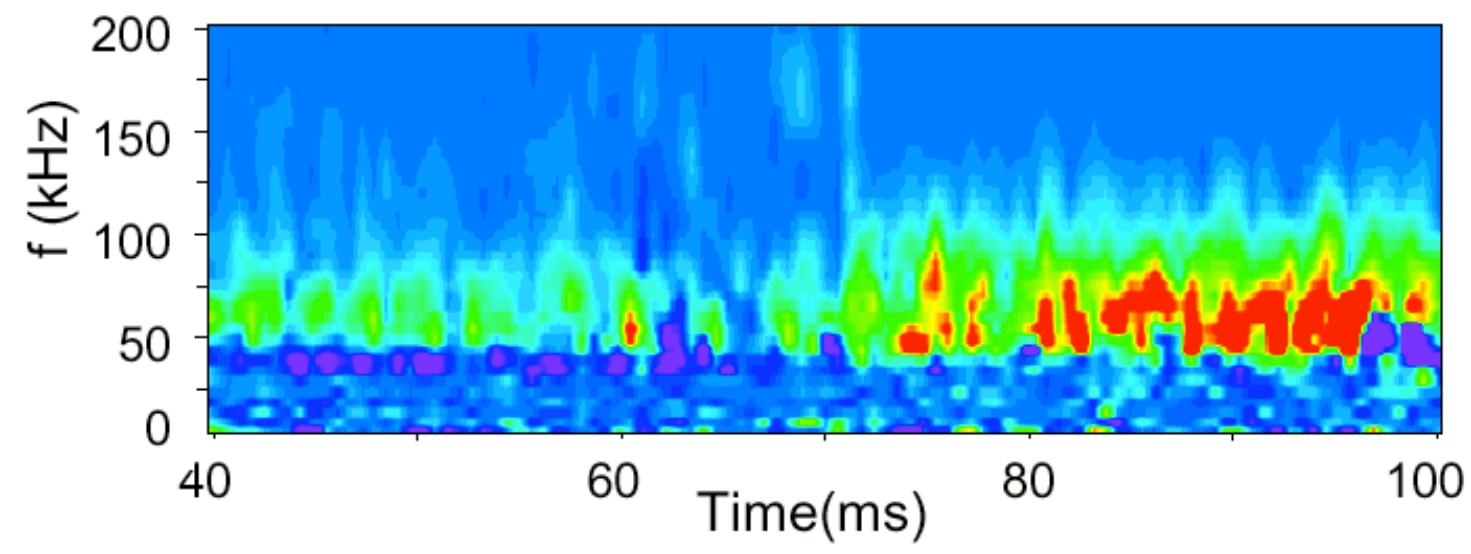

Fig. 7 Time evolution of spectrum of turbulent particle flow with ITB and without ITB. ITB disappeared at $72 \mathrm{msec}$.

This work was conducted in the collaborative research program with the University of Tokyo (NIFS02KZPD003).

\section{References}

[1] A. Fujisawa, et al., Phys. Rev. Lett. 82 (1999) 2669.

[2] T. Minami, et al., Nucl. Fusion 44 (2004) 342.

[3] K. Toi, et al., Plasma Physics and Controlled Nuclear Fusion Research (Proc. Conf. Wurzburg, 1992), IAEA, Vienna (1993) vol. 2, 461.

[4] V. Erckmann. et al., Phys. Rev. Lett. 89 (1993) 2086.

[5] S. Okamura, et al., J. Plasma Fusion Res. 79 (2003) 977.

[6] S. Okamura, et al., Plasma Phys. Control. Fusion 46 (2004) A113.

[7] T. Akiyama, et al., Proc. of 32nd EPS conference, P2-70 (2005)

[8] T. Oishi, et al., Rev. Sci. Instrum. 75 (2004) 4118.

[9] T. Oishi, et al., Proc. of 32nd EPS conference, P4-94 (2005)

[10] A. Fujisawa, et al., Phys. Rev. Lett. 93 (2004) 165002-1. 